is no discussion of the vitally important variations from the classical picture and therefore of the difficulties in diagnosis; the clinical picture of appendicitis in infancy is not mentioned.

The book lacks balance: in numerous sections the common and important conditions in differential diagnosis are either not mentioned, or are mentioned only at the end of the section: faeces follows hydrops of the gall bladder or urachal cyst as a cause of abdominal masses. There are sections on traumatic haemobilia, acute prostatitis, and the calcifying epithelioma of Malherbe, described as a 'fairly common disease'. There is a list of 64 causes of hypoglycaemia, including nesidioblastosis, but the hypoglycaemic troubles of the diabetic are not mentioned. It is remarkable that whereas at least $90 \%$ of childhood symptoms may be the side effects of drugs these are scarcely ever mentioned in differential diagnosis.

I cannot recommend this book.

CYNTHIA M. ILLINGWORTH

\section{Shorter notices}

Increased Intracranial Pressure in Children. Diagnosis and Treatment. Major Problems in Clinical Paediatrics, Volume 8. By W. E. Bell and W. F. McCormick. (Pp. 485; illustrated + tables. $£ 21.00$ hardback.) Holt-Saunders: Eastbourne. 1978.

Elegantly produced, like all the volumes in this series, and well illustrated. Straightforward accounts of hydrocephalus, tumours, etc. Disappointingly vague on some difficult areas like the diagnosis of cerebral oedema, and whether to do a lumbar puncture in the child with suspected increased pressure.

Pediatric Neurology and Neurosurgery. Edited by R. A. Thompson and J. R. Green. (Pp. 420; illustrated + tables. $£ 17.50$ hardback.) SP Medical and Scientific: London. 1978.

Not a textbook, but a collection of reviews of some problems in this field including prenatal diagnosis, migrainous syndrome, some aspects of convulsions including surgery for epilepsy, and rather selective brief chapters on neurosurgery of trauma and tumours. Nothing very new, and no clear indication of the intended audience.

Practical Paediatric Endocrinology. By C. G. D. Brook. (Pp. 142; illustrated + tables. £6.30.) Academic Press: London. 1978.

Although it may be difficult to see who is going to find this 'slim volume' of 129 pages just what they need, those who do read it will discover that the author's potted experience does often provide in a few simple sentences what a larger textbook takes a few pages and much circumlocution to say. At a time when medical texts tend to be painfully overblown, telling the reader more than he wants to know and at far greater length, it is something of a pleasure to find one which tells him, if anything, rather less that he would like. 